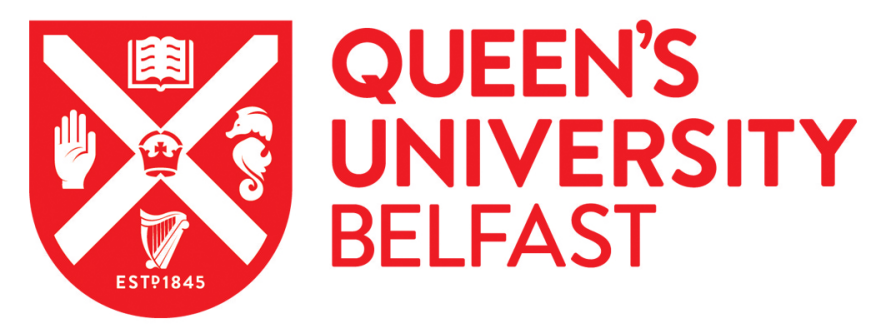

\title{
Magnetic field generation during intense laser channelling in underdense plasma
}

Smyth, A. G., Sarri, G., Vranic, M., Amano, Y., Doria, D., Guillaume, E., Habara, H., Heathcote, R., Hicks, G., Najmudin, Z., Nakamura, H., Norreys, P. A., Kar, S., Silva, L. O., Tanaka, K. A., Vieira, J., \& Borghesi, M. (2016). Magnetic field generation during intense laser channelling in underdense plasma. Physics of Plasmas, 23(6), [063121]. https://doi.org/10.1063/1.4953547

Published in:

Physics of Plasmas

Document Version:

Peer reviewed version

Queen's University Belfast - Research Portal:

Link to publication record in Queen's University Belfast Research Portal

\author{
Publisher rights \\ Copyright 2016 AIP Publishing \\ This article may be downloaded for personal use only. Any other use requires prior permission of the author and AIP Publishing. \\ The following article appeared in Smyth, AG, Sarri, G, Vranic, M, Amano, Y, Doria, D, Guillaume, E, Habara, H, Heathcote, R, Hicks, G, \\ Najmudin, Z, Nakamura, H, Norreys, PA, Kar, S, Silva, LO, Tanaka, KA, Vieira, J \& Borghesi, M 2016, 'Magnetic field generation during \\ intense laser channelling in underdense plasma' Physics of Plasmas, vol 23, no. 6, 063121., 10.1063/1.4953547 and may be found at \\ http://scitation.aip.org/content/aip/journal/pop/23/6/10.1063/1.4953547
}

\section{General rights}

Copyright for the publications made accessible via the Queen's University Belfast Research Portal is retained by the author(s) and / or other copyright owners and it is a condition of accessing these publications that users recognise and abide by the legal requirements associated with these rights.

Take down policy

The Research Portal is Queen's institutional repository that provides access to Queen's research output. Every effort has been made to ensure that content in the Research Portal does not infringe any person's rights, or applicable UK laws. If you discover content in the Research Portal that you believe breaches copyright or violates any law, please contact openaccess@qub.ac.uk. 


\section{Magnetic Field Generation during Intense Laser Channelling in Un derde Thisempysiscrint was accepted by Phys. Plasmas. Click here to see the version of record. A Inderdensempryscrintwa}

Publishifg. Smyth ${ }^{1}$, G. Sarri ${ }^{1}$, M. Vranic ${ }^{2}$, Y. Amano ${ }^{3}$, D. Doria ${ }^{1}$, E. Guillaume ${ }^{2}$, H. Habara ${ }^{3}$, R. Heathcote ${ }^{4}$, G. Hicks ${ }^{5}$, Z. Najmudin ${ }^{5}$, H. Nakamura ${ }^{5}$, P. A. Norreys ${ }^{4}$, S. Kar ${ }^{1}$, L. O. Silva ${ }^{2}$, K. A. Tanaka ${ }^{3}$, J. Vieira $^{2}$, and M. Borghesi ${ }^{1}$

1 School of Mathematics and Physics, The Queen's University of Belfast. University Road, Belfast, BT7 1NN, UK.

2 GoLP/IPFN, Instituto Superior Técnico, Universidade de Lisboa, 1049-001 Lisbon, Portugal

3 Graduate School of Engineering Osaka University. Suita, Osaka, 5650871, Japan.

4 STFC Rutherford Appleton Laboratory. Didcot, Oxon OX1 0Qx, United Kingdom.

5 Blackett Laboratory, Imperial College London. Prince Consort Road, London, SW7 2BZ, United Kingdom.

Channel formation during the propagation of a high-energy (120 J) and long duration (30 ps) laser pulse through an underdense deuterium plasma has been spatially and temporally resolved via means of a proton imaging technique, with intrinsic resolutions of a few $\mu \mathrm{m}$ and a few ps respectively. Conclusive proof is provided that strong azimuthally symmetric magnetic fields with a strength of around $0.5 \mathrm{MG}$ are created inside the channel, consistent with the generation of a collimated beam of relativistic electrons. The inferred electron beam characteristics may have implications for the cone-free fast-ignition scheme of inertial confinement fusion. 


\section{Introduction}

The interaction of an intense laser pulse with low-density plasma is a subject of great Publishinterest, since it sits at the core of many fundamental practical applications, including electron acceleration [1][2][3][4], ion acceleration [5][6] and high energy x-ray generation [7][8]. Another application of particular interest is inertial confinement fusion (ICF) and in particular fast ignition (FI) [9][10].

A suggested method of achieving FI is to send an extended duration intense laser pulse (up to $100 \mathrm{ps)} \mathrm{through} \mathrm{the} \mathrm{coronal} \mathrm{plasma} \mathrm{surrounding} \mathrm{the} \mathrm{pre-compressed} \mathrm{fuel} \mathrm{core,} \mathrm{prior} \mathrm{to}$ the arrival of the ignition pulse. This channelling pulse would 'bore' though the underdense plasma via the ponderomotive force resulting in a voided channel extending across the $\mathrm{mm}$ scale corona. This has the effect of reducing the distance travelled through an attenuating medium by the ignition pulse to reach the critical surface.

The channel forms by the interaction between the charged particles within the plasma and the steep electric field gradient of the channelling pulse. Initially the electrons within the beam's path experience a force directed radially outwards. As the electrons move outward and create a non-neutral charge distribution the ions follow, albeit slightly later, under a sustained ponderomotive drive. This results in a charge voided channel.

In the past a number of experiments have been conducted to study the formation of laser driven channels. Many of these [11][12][13][14][15] have used short pulse durations and/or short observation times ( $\leq 1 \mathrm{ps}$ and $\leq 200 \mu \mathrm{m}$ respectively). Diagnostically, these earlier experiments used a range of techniques including optical interferometric methods and soft Xray radiography.

Many of these previous experiments employed relativistic intensities which can introduce several detrimental non-linear instabilities into the system including filamentation [16][17], self-phase modulation [11][18] and transverse 'hosing' of the laser pulse as it propagates through the plasma [19]. It has been shown [20] that increasing the laser pulse duration can contain and prevent some of these instabilities forming. More recently it has been demonstrated via 2D particle in cell simulations [21] that by sufficiently increasing the duration of the channelling pulse it is possible to create stable $\mathrm{mm}$ scale channels. The simulations were concerned with an electron density range from $0.1 n_{c}$ to $1.02 n_{c}$ where $n_{c}$ is the critical plasma density.

The range simulated by Li et al. [21] covered only a narrow section at the upper end of the coronal density range. Sarri et al. [22] produced stable $\mathrm{mm}$ scale channels over the lower end of the coronal density scale $\left(10^{-4} \mathrm{n}_{\mathrm{c}}<\mathrm{n}_{\mathrm{e}}<10^{-3} \mathrm{n}_{\mathrm{c}}\right)$ recorded using proton radiography. This experiment also recorded the electric field generated across the channel formed by using particle tracing simulations showing that the electric profile was consistent with the channel electron density profile i.e. the electric field peaks were situated at the same positions as the channel's walls where charge has accumulated due to ponderomotive expulsion from inside the channel. Further simulations and experiments have been conducted using similar setups considering the effects of near critical density plasmas [23] and polarisation effects [24] upon channel formation.

Along with electric fields magnetic are also generated during channelling. These fields arise mostly due to the acceleration of electrons within the channel region. A lot of work has went into charactering magnetic fields generated in intense laer-plasma interactions using Faraday rotation, however, these use interaction pulses with duration in the tens of fs [25] [26][27]. Borghesi et al. [28] have made measurements of the magnetic fields generated in similar conditions with an interaction pulse with a duration of a couple of ps. The spatial and temporal evolution of the magnetic fields generated using longer interaction pulse durations (30 ps in work) has never been documented before. Proton radiography provides an excellent 
tool to perform this task with as it enables high resolution measurements to be made in both

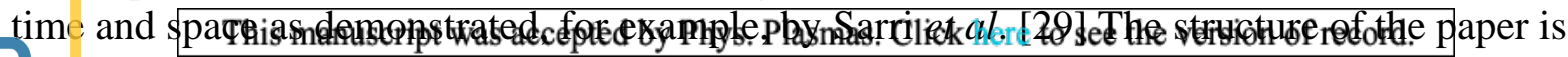
the interaction and section $\mathrm{V}$ discusses the particle tracing analysis conducted to arrive at the conclusions stated in section IV.

\section{Experimental Setup}

The experiment was conducted at the Rutherford Appleton Laboratory (RAL) in Oxfordshire. The VULCAN Nd-glass laser system was used providing two chirped pulse amplified (CPA) [30] beams; one incident upon a supersonic deuterium gas jet (interaction pulse) and one incident upon a $25 \mu \mathrm{m}$ Au foil (probe pulse). Both pulses had a wavelength of $1053 \mathrm{~nm}$. The interaction pulse deposited an energy of $120 \mathrm{~J}$ in a pulse duration of $30 \mathrm{ps}$ onto a focal spot size of $\sim 20 \mu \mathrm{m}$ resulting in a focused intensity of $2 \mathrm{x}^{18} \mathrm{~W} / \mathrm{cm}^{2}$. This pulse was preceded by a lower energy pedestal with a typical intensity contrast of $\sim 10^{-7}$. The probe pulse carried an energy of $60 \mathrm{~J}$ in a duration of 1 ps onto a focal spot size of $\sim 5 \mu \mathrm{m}$ which resulted in an on focus intensity of $2 \times 0^{19} \mathrm{~W} / \mathrm{cm}^{2}$. The probe pulse was focused onto a $25 \mu \mathrm{m}$ thick Au foil. This produced a proton beam via target normal sheath acceleration (TNSA) [31]. The protons had a Maxwellian energy spectrum with a cut-off energy of $\sim 6 \mathrm{MeV}$; this was the highest proton energy recorded.

Figure 1: Schematic diagram of the experimental setup. CPA 1 is the probe pulse that produces the proton beam for radiography. CPA 2 is the interaction pulse that drives the ponderomotice channelling.

As the protons pass through the interaction region they are subject to deflection from the electromagnetic fields present therein. After having passed through the region to be probed the protons are recorded on a stack of Radiochromic film (RCF). The extent and form of the proton density modulation (accumulation or dispersion) is used to infer the strength and form of the fields present in the plasma channel. Another benefit of this technique is that each layer of the RCF stack will stop a different spectral slice of the proton beam implying that each layer of the RCF records a different temporal window of channel evolution. This allows a picture of both the spatial and temporal evolutions of the plasma channel to be obtained [32] with spatial uncertainty of a few $\mu \mathrm{m}$ and a temporal uncertainty of 1-2 ps.

The image observed on the RCF is a magnified projection of the interaction area. The distances between the proton source and the interaction region (i.e. the distance between the $\mathrm{Au}$ foil and the deuterium plasma), 1, and the distance between the interaction region and the detector (i.e. distance between the deuterium plasma and the RCF stack), L, lead to a magnification on the RCF layers given by the equation,

$$
M \approx \frac{L+l}{l}
$$

In the above experiment $\mathrm{L}$ is $\sim 4.5 \mathrm{~cm}$ and 1 is $\sim 0.6 \mathrm{~cm}$. This gives a total magnification of around 8.5 .

\section{Experimental Results}

Figure 2 shows several snapshots of the channel formed in the experiment. The laser pulse propagates from left to right and exits the system at around the time captured by 2(b.). Clear 
con rast can be seen between the lighter regions of the voided channel and the darker regions

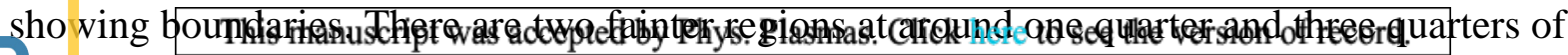
he height of the image. These represent the channel's walls and are consistent with results from previous experiments of this type. In the centre, however, there is a substantially darker Publishing

Figure 2: 3 layers of RCF capturing the interaction at times of (a.) 120 ps, (b.) 70 ps and (c.) 50 ps. The axis on the right hand side displays the co-ordinate system that is used for this and all further images and descriptions.

region which represents a region of field inversion inside the channel as mentioned in Kar et $a l$. [33]. In this case it seems to persist for longer (>120 ps compared to just tens of ps) and is observed at lower intensity $\left(2 \times 10^{18} \mathrm{Wcm}^{-2}\right.$ compared to $\left.1.5 \times 10^{19} \mathrm{Wcm}^{-2}\right)$. This lines constitute a deflection profile which contains information concerning the fields present within the channel. This information can be extracted via the procedure discussed in section V. Comparing the channel radius to the time associated with each layer (calculated from the energy of protons that each layer captures) it is possible to build up a picture of the channel expansion velocity. In this manner the channel expansion is found to occur at a rate of $1.3 \times 10^{5} \mathrm{~ms}^{-1}( \pm 8.5 \%)$ (illustrated in figure 3 ). This is of the same order found previously in similar experiments under similar conditions [22] and seems to confirm that channel expansion is driven solely by plasma pressure.

Figure 3: Channel radius shown as a function of time.

\section{Particle-In-Cell Simulations}

In order to better understand the structure of the electromagnetic fields observed in experiments a series of 2D and 3D particle-in-cell (PIC) simulations have been performed. In order to better understand the structure of the electromagnetic fields observed in experiments a series of 2D and 3D particle-in-cell (PIC) simulations have been performed with OSIRIS [34]. In OSIRIS, electromagnetic fields are stored on a discretised spatial grid. They are advanced in time according to the Maxwell's equations solved at grid points. Plasma particles, however, explore the full 6D phase space. Their motion is directed by the Lorentz force, which is calculated using the electromagnetic fields interpolated from the grid points to the particle positions. After advancing all the particles, charge and current distribution from the plasma are deposited to the grid nodes and used in the Maxwell's equations to advance the electromagnetic fields in the next iteration. An initial current density is recorded on a spatial grid and then Maxwell's equations are solved for the same grid and stored. The fields calculated act upon the charged particles and their motion is calculated based upon the interpolation of the fields' to the particles' positions. The new particle positions are then recorded and Maxwell's equations are solved again for the new charge density distribution.

This process repeats for each time step until the simulation has ran its course.

In a typical simulation, a long laser pulse is focused at the entrance of a flat-density underdense plasma slab, which later results in a channel formation. Since the interaction times are several picoseconds, the ions are expected to move, and therefore all the simulations are performed with mobile ions. Full-scale simulations for this setup, even in 2D, require the usage of high-end state-of-the-art supercomputers because the plasma slab is long ( $\mathrm{mm}$ scale) when compared to the optical laser wavelength ( $\mu \mathrm{m}$ scale) that needs to be fully resolved. Therefore, the main analysis presented here is based on PIC simulations with two spatial dimensions. However, 3D simulations of early channel formation were performed with smaller plasma lengths, and they qualitatively agree with the 2D results. Although at later times the channel expands at a slower pace in $3 \mathrm{D}$, the self-consistent field structure 
(which is relevant for the conclusions that follow) is similar in 2D and 3D. Here we show the

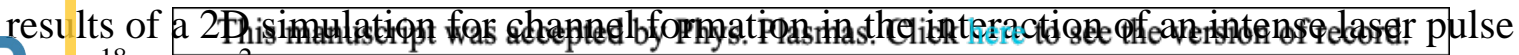
$1 \mathrm{I}=0^{18} \mathrm{~W} / \mathrm{cm}^{2}$ ), with a Gaussian spatial profile, spot-size of $15 \mu \mathrm{m}$ and $5 \mathrm{ps}$ duration (FWHM in the field amplitudes), and a background deuterium plasma $\left(\mathrm{n}_{0}=10^{18} \mathrm{~cm}^{-3}\right)$. A scaled down pulse duration of 5 ps was chosen because running the simulation for a full duration of $>100 \mathrm{ps}$ with a pulse duration of $30 \mathrm{ps}$ was beyond the available

Figure 4: Self-consistent electric field in different times of the channel evolution. Dark blue lines show the position of the laser envelope for reference. (a) The initial stage: the space charge fields are formed mainly by the electron expansion since the ion density distribution is still similar to the original one. (b) Semi-formed channel: the ions are moving and the electric field changes the sign within the channel centre. On the right-hand side we see a nonlinear interaction of the laser peak with the plasma. (c) Fully-formed channel: the electrons and ions have very similar density distribution, which is visible here through a narrow region of space-charge fields on the channel walls. The self-consistent electric field within the channel has smaller amplitude than during the channel formation. $\mathrm{x}_{1}$ and $\mathrm{x}_{2}$ refer to $\mathrm{z}$ and $\mathrm{y}$ axes respectively.

computational capabilities. The channelling laser pulse is linearly polarised in z (out of the simulation plane) direction. The plasma slab is $1.9 \mathrm{~mm}$ long and $0.8 \mathrm{~mm}$ wide, with a flat-top density profile that has a $30 \mu \mathrm{m}$ linear ramp in the $\mathrm{x}$ direction. The simulation box is $2.87 \mathrm{~mm}$ long and $0.8 \mathrm{~mm}$ wide, where in y perpendicular direction the entire simulation box is filled with plasma and uses periodic boundary conditions. The number of cells used is $54000 \mathrm{x}$ 2500 , the timestep 0.16 fs and each cell contains two electrons and two ions. Total simulated time was 21.2 ps. Figure 4 shows the simulated electrostatic field distribution at three separate times. The results here are averaged over the fast-oscillating component of the laser, and therefore the fields presented correspond to the slow-varying fields of the plasma. As the incoming laser propagates through the plasma it radially expels plasma electrons via the ponderomotive force. Since ions take longer than electrons to respond, radial space charge fields appear within the plasma channel. A sheath of high plasma density (2-3 times higher density than the background plasma) surrounds a partially voided channel, which is centred on the laser axis. The space-charge radial electric field within the channel is stronger in the beginning of channel formation than at later times when the ions start to be evacuated. The ponderomotive force will be maximum at the periphery of the laser intensity profile, since there lies the maximum gradient in the square of the electric field. On the other hand, it will be extremely small, if not ideally zero, along the laser propagation axis. We thus expect a region of higher plasma electron density exactly along the laser propagation axis, whose radial oscillations can be clearly seen in Figure 4 (b).

Figure 5: Magnetic field (a-b) and the electric current density (c-d). The azimuthal self-consistent magnetic field surrounds the on-axis current filament made of electrons moving in the same direction as the laser (blue). The reverse current on the channel walls (red) reinforces the strong magnetic field within the channel, while outside of the channel it reduces its amplitude close to zero.

These oscillations are mainly driven by the azimuthal magnetic field shown in Figure 5. When the forward-moving electrons (positive $\mathrm{x}$ direction of propagation gives negative current, blue in Figure 5 (c) and (d)) are displaced radially away from the axis, the magnetic field induces a rotation of their momentum back towards the axis. Therefore, the current filament in the centre of the channel has a stable propagation. The electrons are mainly accelerated directly by the laser field, while the self-consistent fields of the plasma act to keep them in the region of the highest laser amplitude.

The PIC simulations thus provide a clear evidence for strong magnetic fields inside the laser-driven channel. In order to ascertain whether the observed accumulation of probing protons along the main channel axis is due to such magnetic fields, particle tracing 
simulations of the propagation of the probing proton beam through the electromagnetic fields

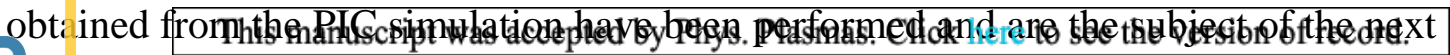

AlPitim

\section{Publishigg Particle Tracing Simulations}

A particle tracing code was used to simulate the trajectories of the probing protons as they passed through the interaction area falling subject to the 3D electromagnetic fields within and produce $2 \mathrm{D}$ proton density maps in the detector plane. Proton trajectories are calculated as they proceed from a virtual point source to a proton detector through a region occupied by electromagnetic fields. The trajectories are calculated by numerically integrating the nonrelativistic equation of motion for the protons as they travel through an area under the effects of an external B field. The interaction between protons within the probe beam is neglected as is the co-moving electron cloud.

Figure 6: Flow chart detailing the process of the of the particle tracer.

The electric field across the channel is measured in other work [22] and shown to strongly resemble that calculated in the PIC simulations. Therefore the electric field used is given the same form as that seen in [22]. This is a radially bi-polar electric field that peaks sharply on both sides of the channel at positions that correspond to the channel walls. The magnetic field is seen from the PIC codes to be more complex. It circulates around the centre of the channel azimuthally. By invoking cylindrical symmetry it becomes clear that in the geometry used the magnetic field observed is comprised only of the $\varphi$ component. This results in a B field defined by the expression,

$$
\boldsymbol{B}_{\boldsymbol{\phi}}=B_{0}\left[e^{\left(\frac{\rho-r_{B}}{L_{r}}\right)^{2}}-e^{\left(\frac{\rho+r_{B}}{L_{r}}\right)^{2}}\right]
$$

Figure 7: Particle tracing y- velocity maps of protons travelling through laser-driven channels for (a.) a collimated probe beam and (b.) a divergent beam.

here $\mathrm{B}_{0}$ is the peak magnetic field, $\rho$ is the radial co-ordinate of the channel, $r_{B}$ is distance between the $\mathrm{z}$-axis and the peak of the field and $\mathrm{L}_{\mathrm{r}}$ is the width (FWHM) of the field. The proton beam that probes these fields is projected from a point-like source and is divergent. This divergence is the reason that the azimuthal field may be probed as a collimated beam would experience no net deflection as it passed through the channel region. As the protons pass through the channel region they would experience a force with a direction determined by the angle they form with the magnetic field. With a collimated beam this force is equal and opposite on one side of the channel as that on the other. A proton from a divergent beam, however, possesses an initial trajectory with non-zero $\hat{\mathbf{y}}$ and $\hat{\mathbf{z}}$ components. This means that the forces experienced on one side of its transit through the channel region are not undone by the experienced on the other. This results in a net proton velocity shift based on angular trajectory. Figure 7 (a.) shows the velocity shift map for collimated protons passing through the channel region. Only protons passing the boundaries of the channel are shifted. This is because they only experience the field in a single direction. This translates as a downward shift on the bottom of the channel and an upward shift on the top with protons passing through the centre experiencing no net shift. Figure 7 (b.) show the same map but for a divergent beam. There are velocity shifting regions inside the channel this time, however, there is a reversal of shifting direction when the $\mathrm{z}=0$ point is crossed. This corresponds to 
the centre of the probe beam. Protons on the right hand side of the beam are seen to be

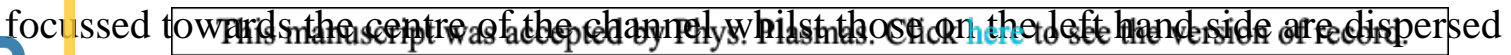

Alp ut wards towards the boundaries. The case presented in Figure 7 (b.) agrees with what is expected from PIC simulations (Figure 8).

Publishing Using expressions for the electric and magnetic fields described above particle tracing simulations were ran to determine the values for the field parametres present in the data. Using the relative modulation of the proton beam as it crossed the channel, $\delta n_{p} / n_{p 0}$, particle tracing simulations were compared to the experimental data (Figure 9). A very good agreement was reached for the $2^{\text {nd }}$ layer of the RCF (i.e $\sim 120 \mathrm{ps)}$. The electric field was found to peak at $\sim 160 \mu \mathrm{m}$ from the centre of the channel with a magnitude of $2 \times 10^{7} \mathrm{Vm}^{-1}$. The magnetic field had a peak magnitude, $B_{0}$, of $0.40 \pm 0.05 \mathrm{MG}$ whilst $\mathrm{r}_{\mathrm{B}}$ and $\mathrm{L}_{\mathrm{r}}$ had values of $70 \mu \mathrm{m} \pm 12.3 \%$ and $65 \mu \mathrm{m} \pm 14.4 \%$ respectively. By repeating this process for the other layers it is possible to build up a picture of the magnetic field's temporal evolution. Overlaying this with the evolution predicted by the PIC simulations reveals a high level of agreement between the two (Figure 10.). A second shot captured with an aperture removed revealed much more detail of the early period of the channel expansion (between 1-2 pulse durations). It shows remarkably similar trends to those observed in Figure 9 (Figure 11). As the PIC simulations were scaled down temporally (as mentioned above) the $\mathrm{x}$-axis of Figure 10 (a.), (b.) and (c.) are relative to the relevant pulse duration (30 ps for the particle tracings and $5 \mathrm{ps}$ for the PIC simulations).(a.), (b.) and (c.) are relative to the relevant pulse duration (30 ps for the particle tracings and 5 ps for the PIC simulations).

Figure 8: Particle trace for protons using the same field values found for the $2.9 \mathrm{MeV}$ (2nd) layer of the RCF stack i.e. $\mathrm{E}=2 \times 10^{7} \mathrm{Vm}^{-1}, \mathrm{~B}=0.50 \mathrm{MG}$.

Figure 9: Relative modulation of the density of the probing proton beam across the laser-driven channel (y-axis). Solid blue line represents the experimental data while the dashed red line is obtained from particle tracing simulations. This match was for the $2^{\text {nd }}$ Layer of the RCF stack.

The electron density within the voided channel is $\sim 0.01$ that of the unmodulated plasma giving a value of order $10^{16} \mathrm{~cm}^{-3}$. The electron temperature is $T_{\mathrm{e}} \sim 7 \times 10^{7} \mathrm{~K}$. These values result in an electron-electron collision frequency of $v_{\mathrm{ee}} \sim 1.25 \times 10^{6} \mathrm{~Hz}$ and an electron conductivity of $\sigma_{\mathrm{e}} \sim 5.6 \times 10^{8} \mathrm{Sm}^{-1}$. Using these values and using the radius of the channel $(\sim 100 \mu \mathrm{m})$ as the magnetic field scale length the diffusion time scale of the field can be estimated at $\tau_{D} \sim 2 \mu \mathrm{s}$. This indicates that the magnetic field does not fade due to diffusion over the course of the observation, however, it is seen to evolve (Figure 10). Comparing Figure 10 (a.) and (c.) shows roughly antithetical evolutions with $B_{0} \propto 1 / L_{r}$. Given the Gaussian nature of the magnetic field and assuming that there is no significant energy loss in the system then a reasonable approximation is $B^{2} \times{L_{r}}^{2} \approx$ constant. This is largely seen to be the case from Figure 10 and so the major evolution of the magnetic field is driven simply by the expansion of the channel and the stretching of the magnetic field within.

Figure 10: The PIC simulation results (solid blue) and the particle traced data (red data points) plotted together for (a.) $B,(b.) r_{B}$ and (c.) $L_{r}$.

Figure 11: Magnetic field against time in pulse durations for the early period of channel expansion. The nature of the trend observed matches with the PIC simulation between 1-2 pulse durations.

The simulations indicate that the magnetic field is generated by an electron current that runs along the centre of the channel. This concurs with the beam shown along the channel axis from the particle tracings. As discussed in section 3 laser driven channels are formed by pondermotive expulsion of charge lying within the laser's path. Many of the electrons that are 
not radially expelled experience direct laser acceleration (DLA)[35] along the $\mathbf{z}$ direction.

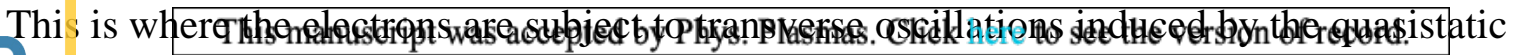

A Clectric and magnetic fields induced within the channel. The laser's electric field then accelerates the electrons that are 'wiggling' within the channel's fields leading to a beam of refativistic electrons. By geometric consideration eqn. 2 is the $\boldsymbol{\theta}$ component of the magnetic field in cylindrical co-ordinates $(\boldsymbol{\rho}, \boldsymbol{\phi}, \mathbf{z})$. The curl of $\mathbf{B}$ then reduces to,

$$
\boldsymbol{\nabla} \times \boldsymbol{B}=\frac{1}{\rho}\left(\frac{\partial\left(\rho \boldsymbol{B}_{\varphi}\right)}{\partial \rho}\right) \hat{\boldsymbol{z}}
$$

This enables the simple calculation for the current density, $\mathbf{J} \sim 1.5 \times 10^{12} \mathrm{Am}^{-2} \underline{\hat{z}}$ along the centre of the channel that is consistent with a collimated electron current flowing along $\mathbf{z}$. Assuming relativistic electrons (i.e. $\mathrm{v} \sim \mathrm{c}$ ) the electron density corresponding to $\mathbf{J}$ is, $\mathrm{n}_{\mathrm{e}}=$ $3 \times 10^{16} \mathrm{~cm}^{-3}$. The current density also gives a current of I $\sim 7.5 \mathrm{kA}$ using the FWHM of $\mathbf{J}$ as the cross-section of flow.

\section{Conclusion}

Presented here are the first measurements of the magnetic field produced during laser driven channel formation, using a long (30 ps) pulse, with a uniform density plasma $\left(\mathrm{n}_{\mathrm{e}}\right.$ $10^{18} \mathrm{~cm}^{-3}$ ) conducted at the VULCAN laser facility. The magnetic field is shown to arise from an electron current which is established along the centre of the channel. The magnetic field is seen to persist within the plasma for a relatively long time after the current has died. Values for magnetic field parameters were obtained by conducting particle tracing analysis matched against radiochromic films and backed up by 2D PIC simulations.

It was seen that a peak magnetic field of $0.5 \mathrm{MG}$ was achieved $\sim 70$ ps into the interaction. Using the trapped magnetic field it was found that a peak relativistic electron current of $\sim 15$ kA with an electron density of $\sim 10^{16} \mathrm{~cm}^{-3}$ was produced along the centre of the channel. PIC simulations suggest that this current was produced mainly via direct laser acceleration of electrons.

This electron current may be detrimental towards the process's use in future FI reactions. The electron current is too weak for ignition and so may instead simply heat fuel. A potential solution to this problem may lie with the polarisation of laser radiation used in channel production. Comparing available data between channels created using linearly and circularly polarised laser pulses suggests that the production of the $\mathbf{z}$ electrons is inhibited by the use of circular polarisation. However the nature of the channel produced by a circularly polarised pulse may not be suitable, for the purpose of ICF, when compared to the linearly polarised case [24]. This may form the basis for some future work into the hole-boring procedure.

\section{Acknowledgments}

The authors acknowledge support from the Department and Education and Learning (NI) and from EPSRC (grantEP/I029206/1). This project has also received funding from the European Union's Horizon 2020 research and innovation programme under grant agreement number 633053. The views and opinions expressed herein do not necessarily reflect those of the European Commission.

The authors also gratefully acknowledge the support of the staff of the Central Laser Facility, Rutherford Appleton Laboratory. The work was supported by the United 
Kingdom's Science and Technology Facilities Council and the Engineering and Physical

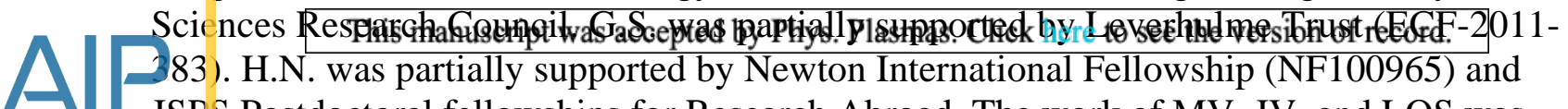
JSPS Postdoctoral fellowships for Research Abroad. The work of MV, JV, and LOS was sugported by the European Research Council (ERC-2010-AdG Grant No. 267841), and Fundação para a Ciência e a Tecnologia (Portugal), under grants PTDC/FIS/111720/2009 and SFRH/BD/62137/2009. We acknowledge PRACE for awarding access to the supercomputers Jügene and SuperMUC based in Germany at Jülich and Leibniz research centers.

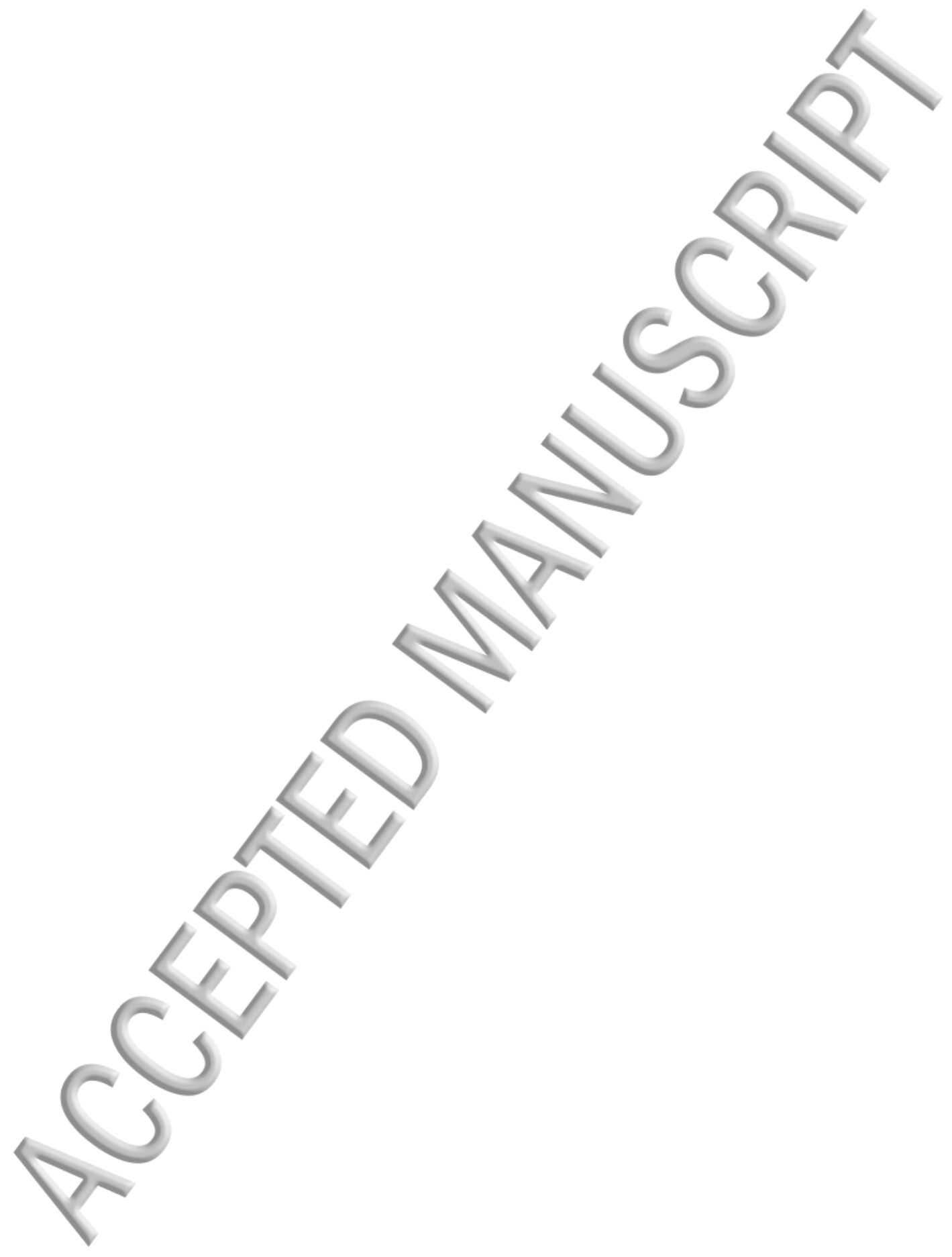




\section{References}

$A \mid R_{11}$

Publishing

[2] W. P. Leemans, B. Nagler, a. J. Gonsalves, C. Tóth, K. Nakamura, C. G. R. Geddes, E. Esarey, C. B. Schroeder, and S. M. Hooker, "GeV electron beams from a centimetre-scale accelerator," Nat. Phys., vol. 2, no. 10, pp. 696-699, Sep. 2006.

[3] E. Esarey, C. Schroeder, and W. Leemans, "Physics of laser-driven plasma-based electron accelerators," Rev. Mod. Phys., vol. 81, no. 3, pp. 1229-1285, Aug. 2009.

[4] V. Malka, J. Er, Y. A. Gauduel, E. Lefebvre, A. Rousse, and K.I. M. T. A. Phuoc, "Principles and applications of compact laser - plasma accelerators," vol. 4, no. June, 2008.

[5] L. Willingale, S. Mangles, P. Nilson, R. Clarke, a. Dangor, M. Kaluza, S. Karsch, K. Lancaster, W. Mori, Z. Najmudin, J. Schreiber, a. Thomas, M. Wei, and K. Krushelnick, "Collimated Multi-MeV Ion Beams from High-Intensity Laser Interactions with Underdense Plasma," Phys. Rev. Lett., vol. 96, no. 24, p. 245002, Jun. 2006.

[6] P. McKenna, K. Ledingham, J. Yang, L. Robson, T. McCanny, S. Shimizu, R. Clarke, D. Neely, K. Spohr, R. Chapman, R. Singhal, K. Krushelnick, M. Wei, and P. Norreys, "Characterization of proton and heavier ion acceleration in ultrahigh-intensity laser interactions with heated target foils," Phys. Rev. E, vol. 70, no. 3, p. 036405, Sep. 2004.

[7] A. Rousse, K. Phuoc, R. Shah, A. Pukhov, E. Lefebvre, V. Malka, S. Kiselev, F. Burgy, J.-P. Rousseau, D. Umstadter, and D. Hulin, "Production of a keV X-Ray Beam from Synchrotron Radiation in Relativistic Laser-Plasma Interaction," Phys. Rev. Lett., vol. 93, no. 13, p. 135005, Sep. 2004.

[8] S. Kneip, C. McGuffey, J. L. Martins, S. F. Martins, C. Bellei, V. Chvykov, F. Dollar, R. Fonseca, C. Huntington, G. Kalintchenko, a. Maksimchuk, S. P. D. Mangles, T. Matsuoka, S. R. Nagel, C. a. J. Palmer, J. Schreiber, K. T. Phuoc, a. G. R. Thomas, V. Yanovsky, L. O. Silva, K. Krushelnick, and Z. Najmudin, "Bright spatially coherent synchrotron X-rays from a table-top source," Nat. Phys., vol. 6, no. 12, pp. 980-983, Oct. 2010.

[9] S. Atzeni, "Inertialfusion fast ignitor: Igniting pulse parameter window vs the penetration depth of the heating particles and the density of the precompressed fuel," Phys. Plasmas, vol. 6, no. 8, pp. 3316-3326, 1999.

[10] M. Tabak, D. S. Clark, S. P. Hatchett, M. H. Key, B. F. Lasinski, R. a. Snavely, S. C. Wilks, R. P. J. Town, R. Stephens, E. M. Campbell, R. Kodama, K. Mima, K. a. Tanaka, S. Atzeni, and R. Freeman, "Review of progress in Fast Ignition," Phys. Plasmas, vol. 12, no. 5, p. 057305, 2005.

[11] M. Borghesi, a. MacKinnon, L. Barringer, R. Gaillard, L. Gizzi, C. Meyer, O. Willi, a. Pukhov, and J. Meyer-ter-Vehn, "Relativistic Channeling of a Picosecond Laser Pulse in a Near-Critical Preformed Plasma," Phys. Rev. Lett., vol. 78, no. 5, pp. 879882, Feb. 1997.

[12] J. Fuchs, G. Malka, J. Adam, F. Amiranoff, S. Baton, N. Blanchot, a. Héron, G. Laval, J. Miquel, P. Mora, H. Pépin, and C. Rousseaux, "Dynamics of Subpicosecond Relativistic Laser Pulse Self-Channeling in an Underdense Preformed Plasma," Phys. Rev. Lett., vol. 80, no. 8, pp. 1658-1661, Feb. 1998.

[13] K. a Tanaka, H. Hashimoto, R. Kodama, K. Mima, Y. Sentoku, and K. Takahashi, "Performance comparison of self-focusing with 1053- and 351-nm laser pulses.," 
Phys. Rev. E. Stat. Phys. Plasmas. Fluids. Relat. Interdiscip. Topics, vol. 60, no. 3, pp. 3283-\$hi§eplandse9pt was accepted by Phys. Plasmas. Click here to see the version of record.

Z. Najmudin, K. Krushelnick, M. Tatarakis, E. L. Clark, C. N. Danson, V. Malka, D. Neely, M. I. K. Santala, and a. E. Dangor, "The effect of high intensity laser propagation instabilities on channel formation in underdense plasmas," Phys. Plasmas, vol. 10, no. 2, p. 438, 2003.

[15] K. Takahashi, R. Kodama, K. Tanaka, H. Hashimoto, Y. Kato, K. Mima, F. Weber, T. Barbee, and Da Silva LB, "Laser-hole boring into overdense plasmas measured with soft X-Ray laser probing," Phys. Rev. Lett., vol. 84, no. 11, pp. 2405-8, Mar. 2000.

[16] W. L. K. S. Wilks, P.E. Young, J. Hammer, M. Tabak, "Spreading of Intense Laser Beams Due to Filamentation," Phys. Rev. Lett., vol. 73, no. 22, pp. 2994-2997, 1994.

[17] N. Naseri, S. G. Bochkarev, and W. Rozmus, "Self-channelling of relativistic laser pulses in large-scale underdense plasmas," Phys. Plasmas, vol. 17, no. 3, p. 033107, 2010.

[18] N. E. Andreev, L. M. Gorbunov, V. I. Kirsanov, and A. S. Sakharov, "Self-modulation of high-intensity laser pulses in underlense plasmas and plasma channels," in AIP Conference Proceedings, 1997, vol. 396, pp. 61-74.

[19] M. C. Kaluza, S. P. D. Mangles, a. G. R. Thomas, Z. Najmudin, a. E. Dangor, C. D. Murphy, J. L. Collier, E. J. Divall, P. S. Foster, C. J. Hooker, a. J. Langley, J. Smith, and K. Krushelnick, "Observation of a Long-Wavelength Hosing Modulation of a High-Intensity Laser Pulse in Underdense Plasma," Phys. Rev. Lett., vol. 105, no. 9, p. 095003, Aug. 2010.

[20] P. E. Young, M. E. Foord, J. H. Hammer, W. L. Kruer, M. Tabak, “Time-Dependent Channel Formation in a Laser-Produced Plasma," Phys. Rev. Lett., vol. 75, no. 6, pp. 1082-1085, 1995.

[21] G. Li, R. Yan, C. Ren, T.-L. Wang, J. Tonge, and W. Mori, "Laser Channeling in Millimeter-Scale Underdense Plasmas of Fast-Ignition Targets," Phys. Rev. Lett., vol. 100, no. 12, p. 125002, Mar. 2008.

[22] G. Sarri, K. L. Lancaster, R. Trines, E. L. Clark, S. Hassan, J. Jiang, N. Kageiwa, N. Lopes, R. Ramis, a. Rehman, X. Ribeyre, C. Russo, R. H. H. Scott, T. Tanimoto, M. Temporal, M. Borghesi, J. R. Davies, Z. Najmudin, K. a. Tanaka, M. Tatarakis, and P. a. Norreys, "Creation of persistent, straight, $2 \mathrm{~mm}$ long laser driven channels in underdense plasmas," Phys. Plasmas, vol. 17, no. 11, p. 113303, 2010.

[23] L. Willingale, P. M. Nilson, a. G. R. Thomas, S. S. Bulanov, a. Maksimchuk, W. Nazarov, T. C. Sangster, C. Stoeckl, and K. Krushelnick, "High-power, kilojoule laser interactions with near-critical density plasma," Phys. Plasmas, vol. 18, no. 5, p. $056706,2011$.

[24] D. K. Singh, J. R. Davies, G. Sarri, F. Fiuza, and L. O. Silva, "Dynamics of intense laser propagation in underdense plasma: Polarization dependence," Phys. Plasmas, vol. 19 , no. 7 , p. $073111,2012$.

[25] M. C. Kaluza, H.-P. Schlenvoigt, S. P. D. Mangles, a. G. R. Thomas, a. E. Dangor, H. Schwoerer, W. B. Mori, Z. Najmudin, and K. M. Krushelnick, "Measurement of Magnetic-Field Structures in a Laser-Wakefield Accelerator," Phys. Rev. Lett., vol. 105, no. 11, p. 115002, Sep. 2010.

[26] B. Walton, a E. Dangor, S. P. D. Mangles, Z. Najmudin, K. Krushelnick, a G. R. Thomas, S. Fritzler, and V. Malka, "Measurements of magnetic field generation at ionization fronts from laser wakefield acceleration experiments," New J. Phys., vol. 15, no. 2, p. 025034, Feb. 2013.

[27] a. Flacco, J. M. Rax, and V. Malka, "Reconstruction of polar magnetic field from single axis tomography of Faraday rotation in plasmas," Phys. Plasmas, vol. 19, no. 
$10,2012$.

$A \mid P^{2 x}$

Jun. 1998

G. Sarri, a. Macchi, C. a. Cecchetti, S. Kar, T. V. Liseykina, X. H. Yang, M. E.

Dieckmann, J. Fuchs, M. Galimberti, L. a. Gizzi, R. Jung, I. Kourakis, J. Osterholz, F.

Pegoraro, a. P. L. Robinson, L. Romagnani, O. Willi, and M. Borghesi, "Dynamics of Self-Generated, Large Amplitude Magnetic Fields Following High-Intensity Laser Matter Interaction," Phys. Rev. Lett., vol. 109, no. 20, p. 205002, Nov. 2012.

[30] D. Strickland and G. Mourou, "Compression of amplified chirped optical pulses," Optics Communications, vol. 55. pp. 447-449, 1985.

[31] A. Macchi, I. Nazionale, M. Borghesi, M. Passoni, and D. Energia, "Ion Acceleration by Superintense Laser Pulses : from Classic Problems to Advanced Applications."

[32] G. Sarri, C. a Cecchetti, L. Romagnani, C. M. Brown, D. J. Hoarty, S. James, J. Morton, M. E. Dieckmann, R. Jung, O. Willi, S. V Bulanov, F. Pegoraro, and M. Borghesi, "The application of laser-driven proton beams to the radiography of intense laser-hohlraum interactions," New J. Phys., vol. 12, no. 4, p. 045006, Apr. 2010.

[33] S. Kar, M. Borghesi, C. a Cecchetti, L. Romagnani, F. Ceccherini, T. V Liseykina, a Macchi, R. Jung, J. Osterholz, O. Willi, L. a Gizzi, a Schiavi, M. Galimberti, and R. Heathcote, "Dynamics of charge-displacement channeling in intense laser-plasma interactions," New J. Phys., vol. 9, no. 11, pp. 402-402, Nov. 2007.

[34] R. A. Fonseca, J. Vieira, F. Fiuza, A. Davidson, F. S. Tsung, and W. B. Mori, "Exploiting multi-scale parallelism for large scale numerical modelling of laser wakefield accelerators," Plasma Phys. Control. Fusion, vol. 124011, no. 55, 2013.

[35] C. Gahn, G. Tsakiris, a. Pukhov, J. Meyer-ter-Vehn, G. Pretzler, P. Thirolf, D. Habs, and K. Witte, "Multi-MeV Electron Beam Generation by Direct Laser Acceleration in High-Density Plasma Channels," Phys. Rev. Lett., vol. 83, no. 23, pp. 4772-4775, Dec. 1999. 


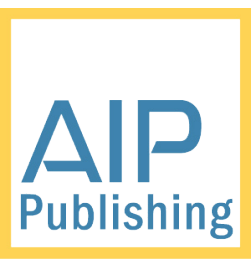

This manuscript was accepted by Phys. Plasmas. Click here to see the version of record.

CPA 1

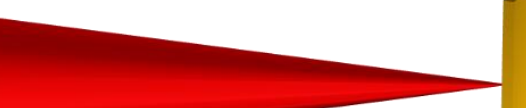

$25 \mu \mathrm{m}$

Au Foil $1=0.6 \mathrm{~cm}$ $\mathrm{L}=4.5 \mathrm{~cm}$

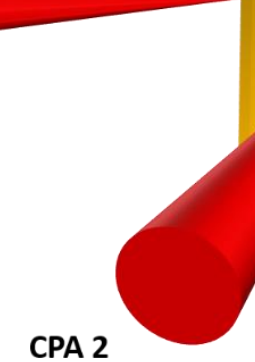

CPA 2

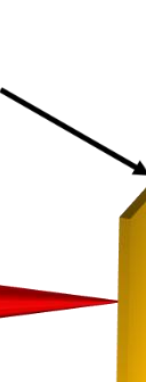

$\stackrel{1}{1}$

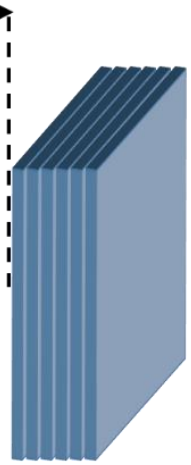

Multi-layer

RCF Stack 


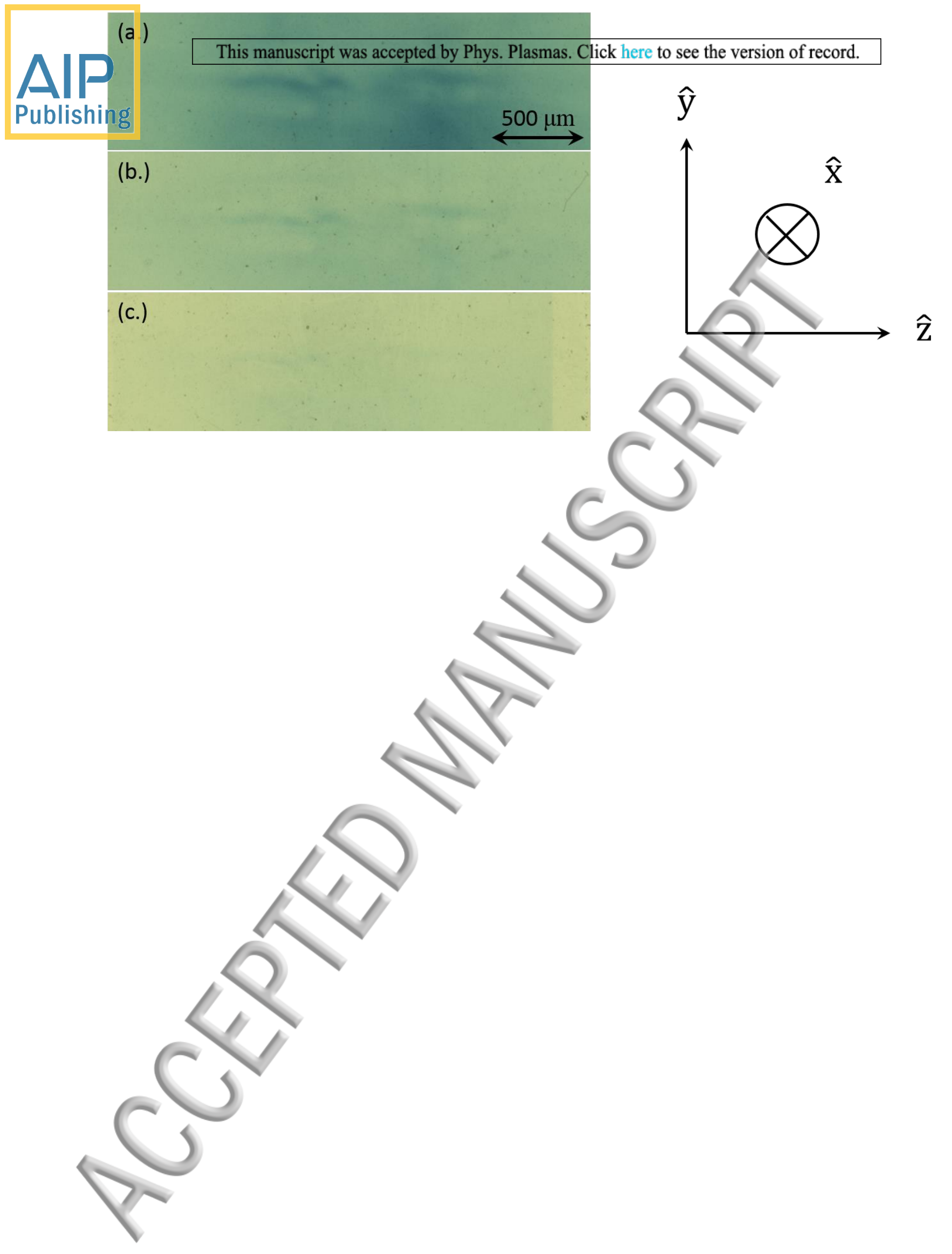




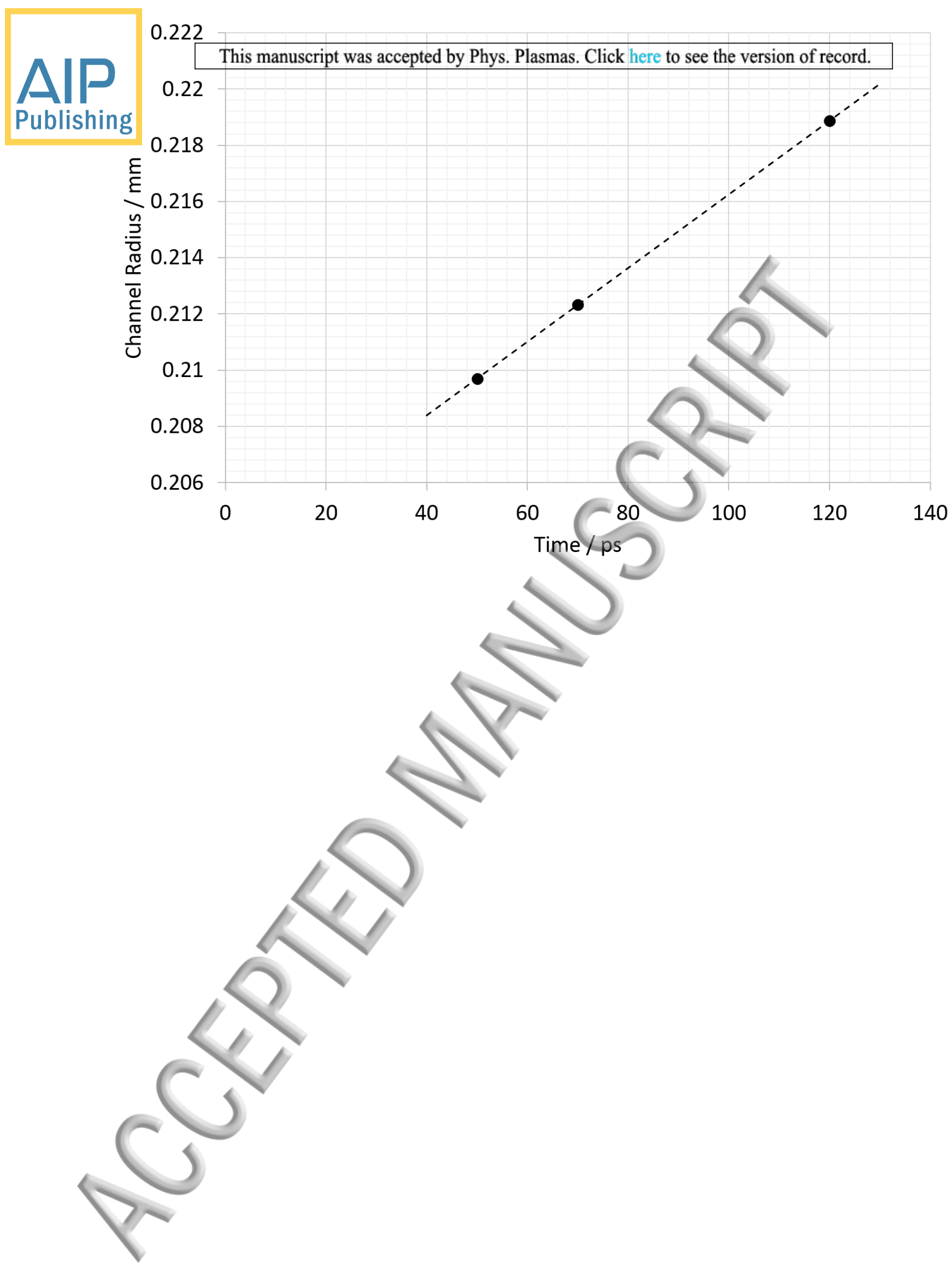



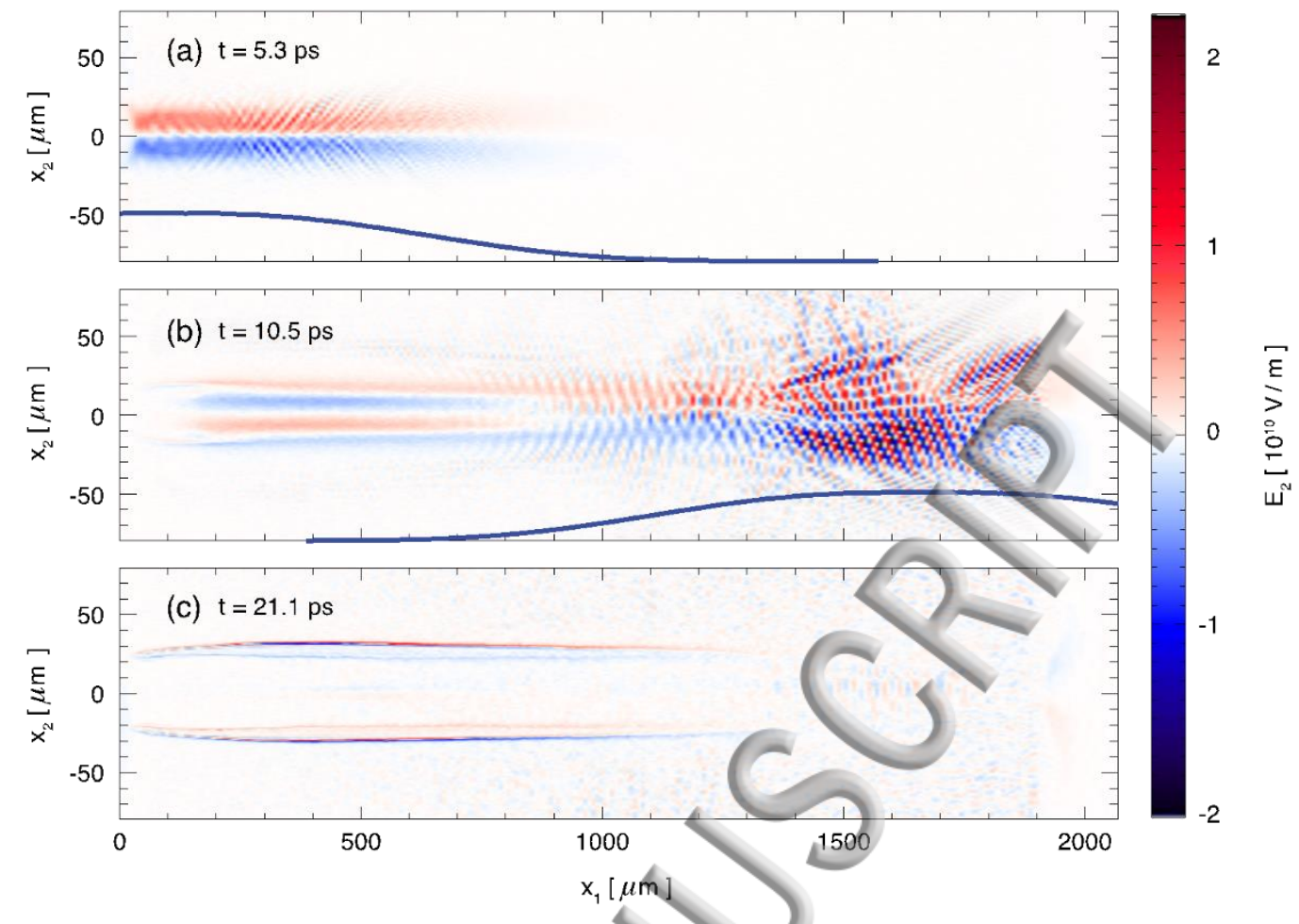


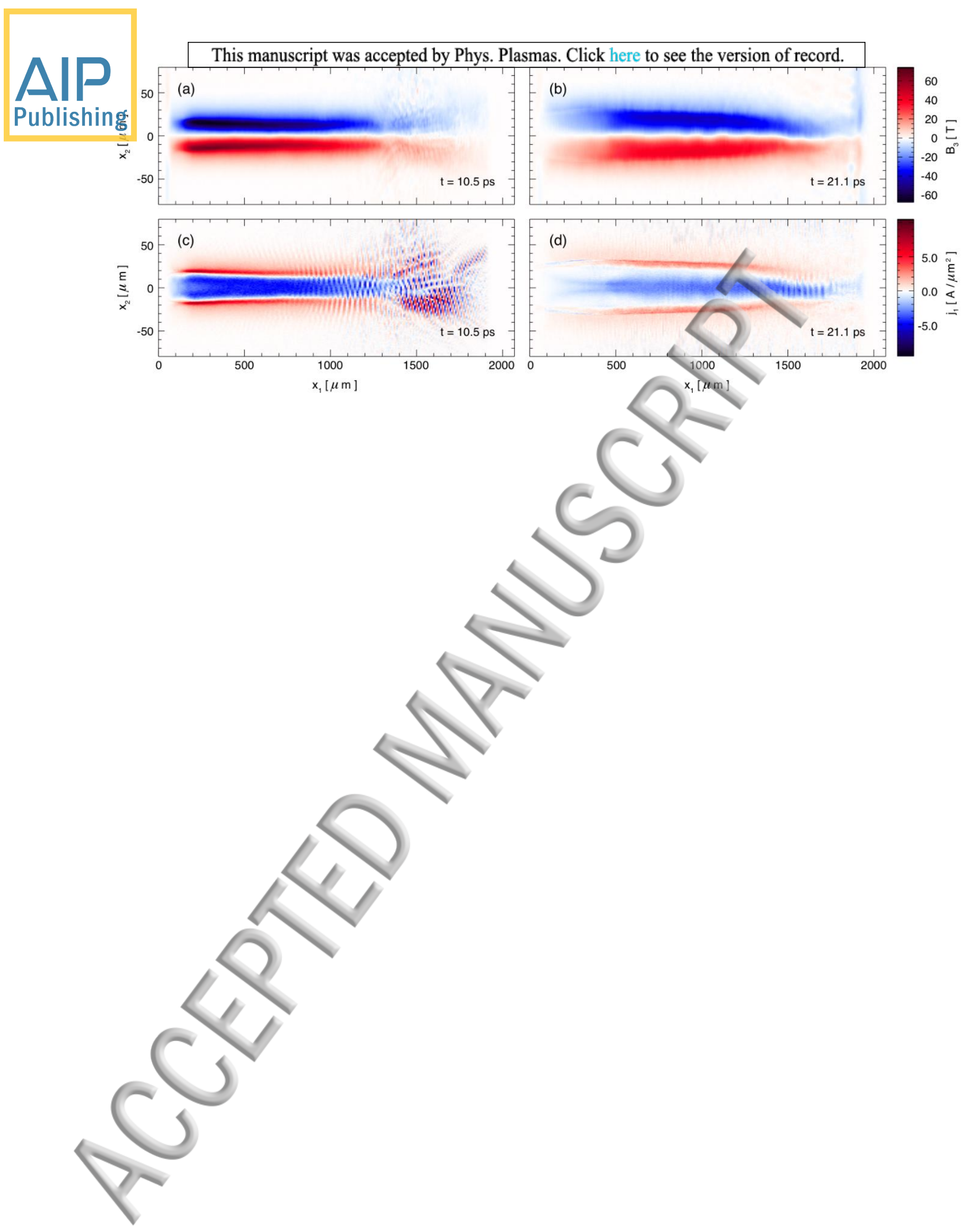



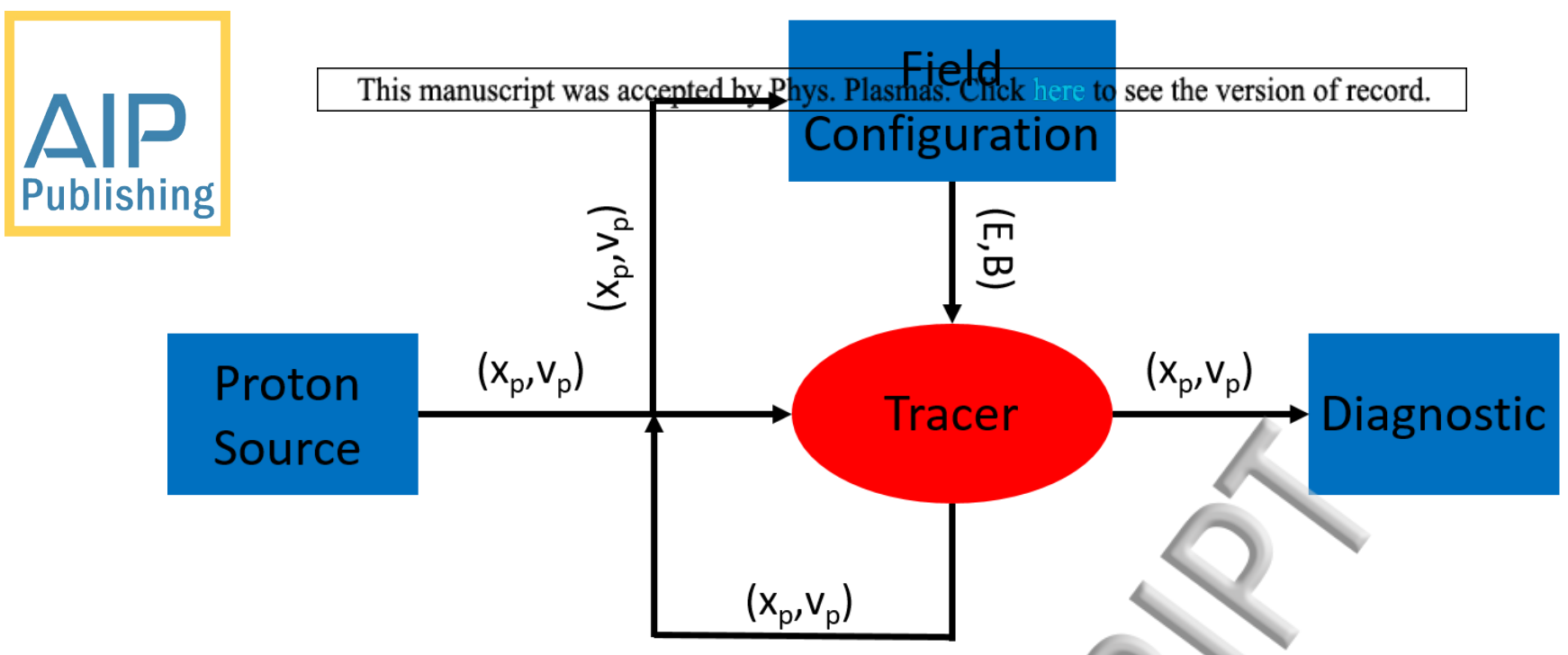


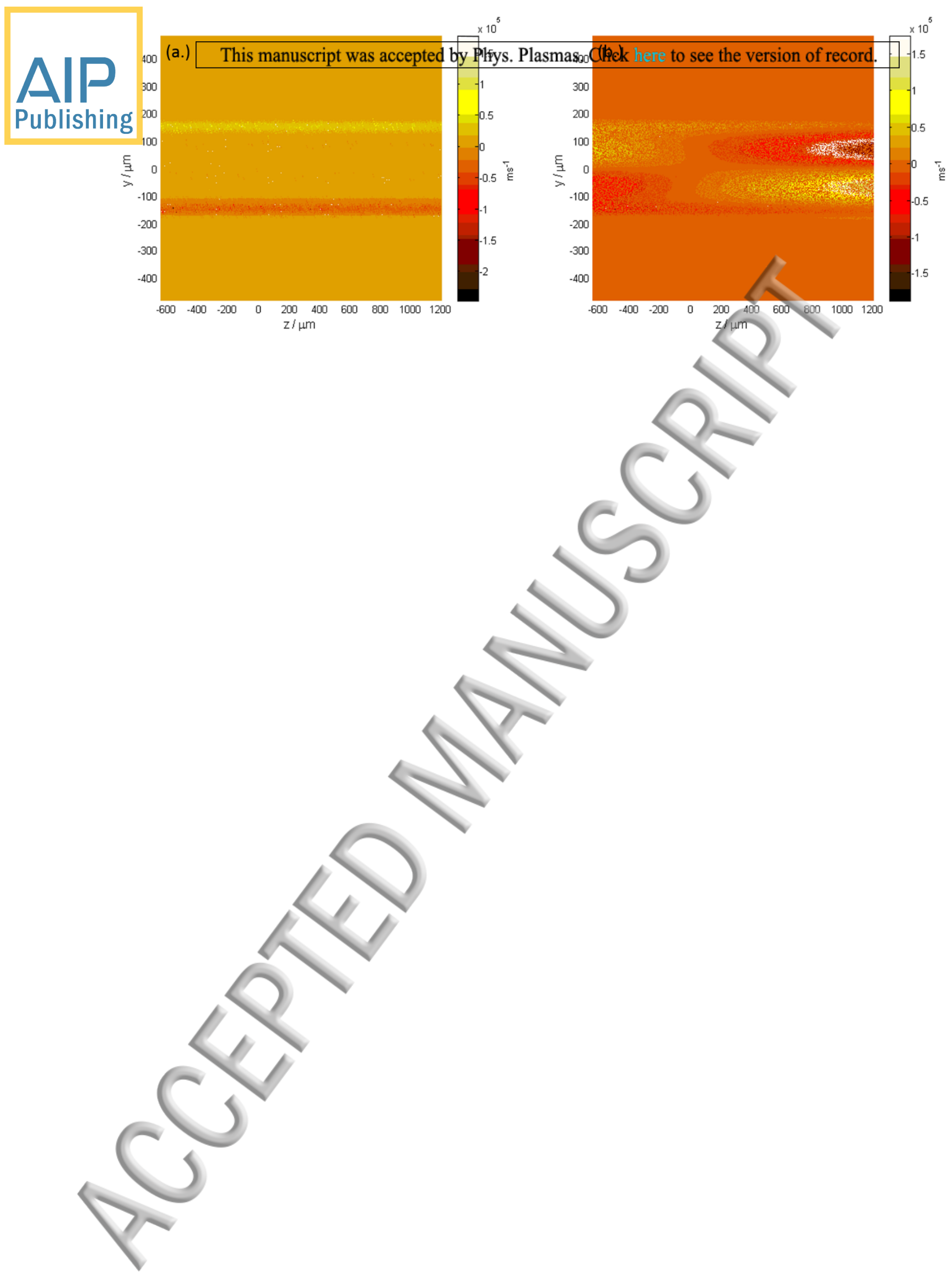




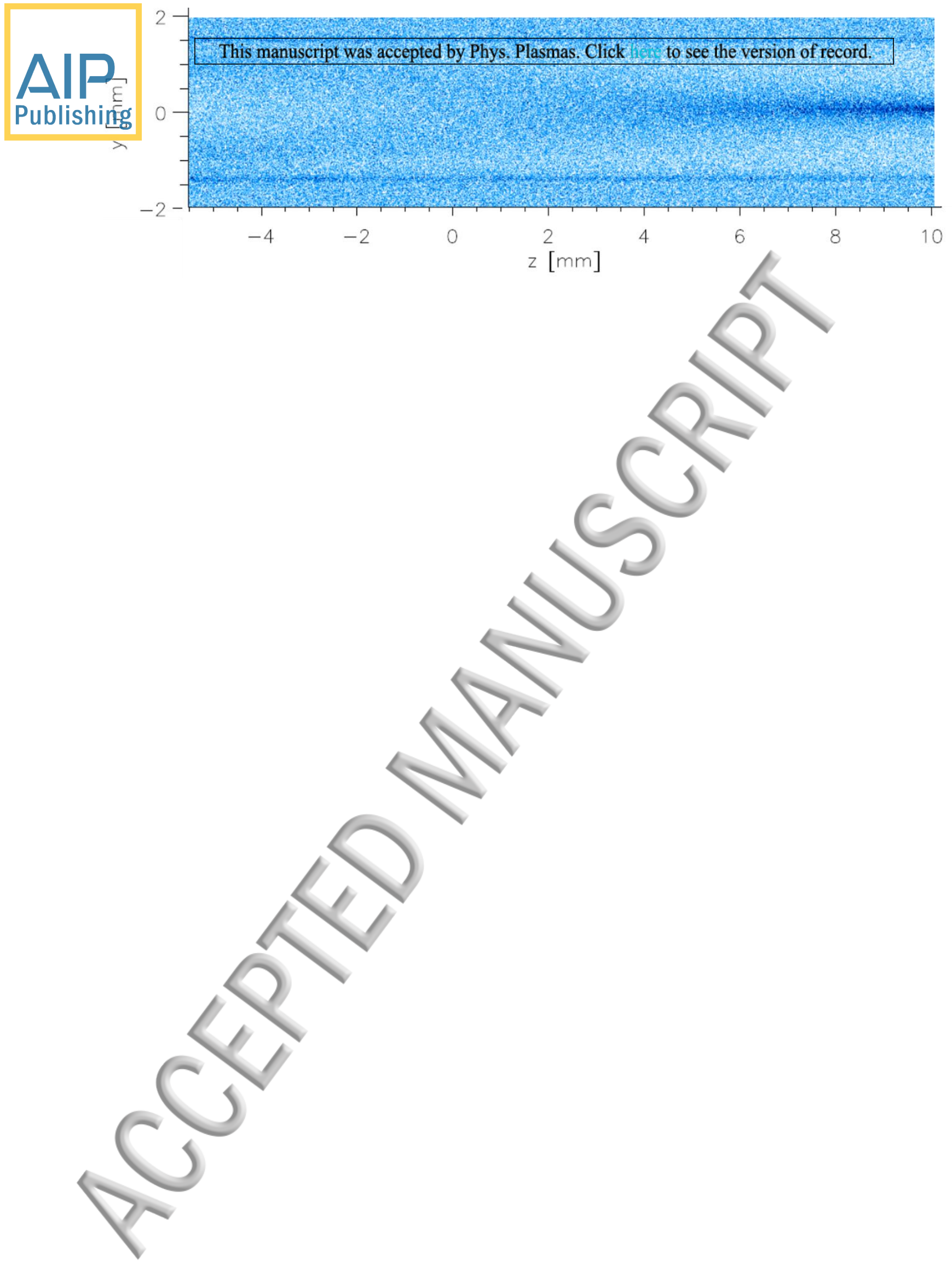




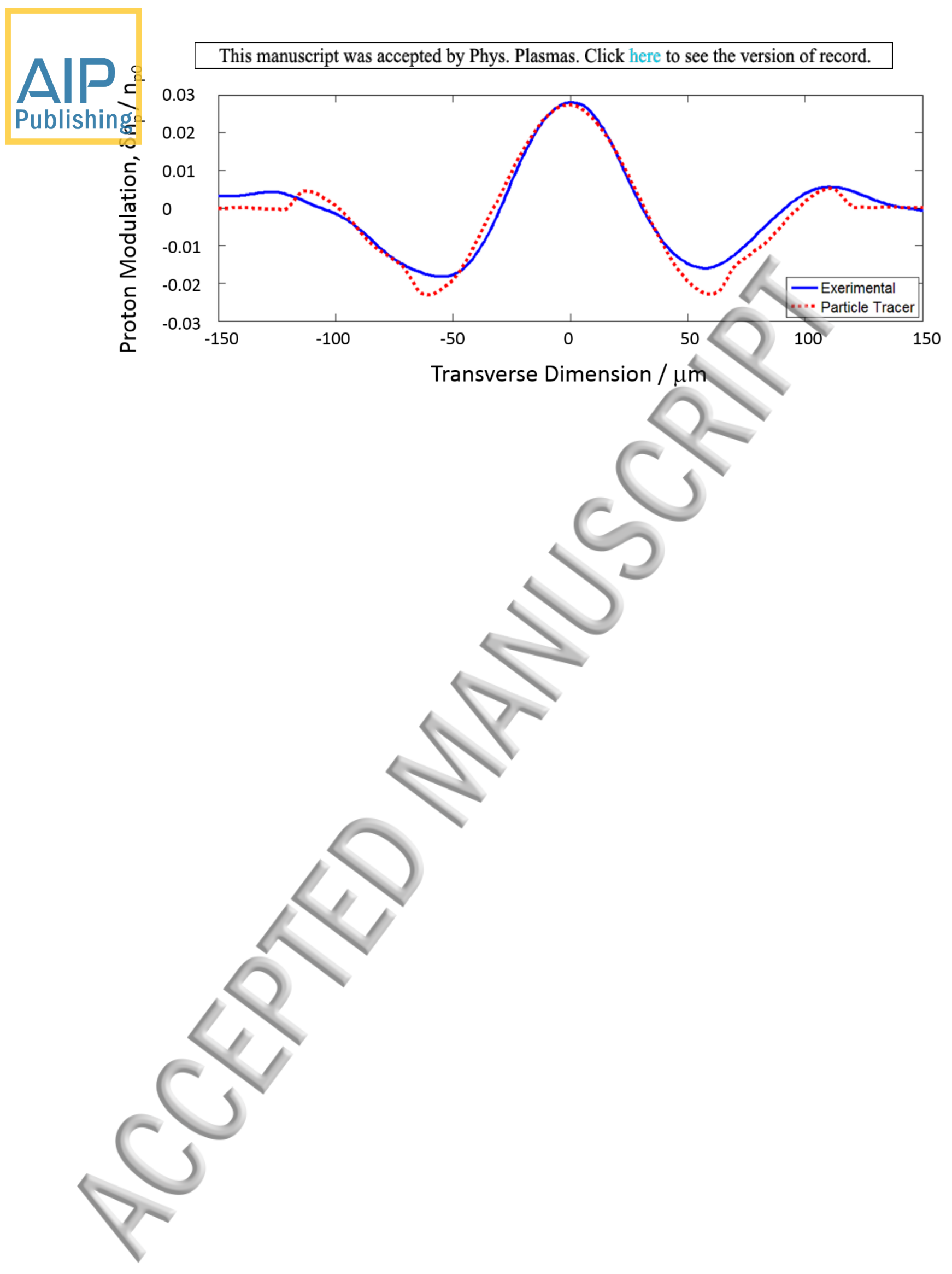




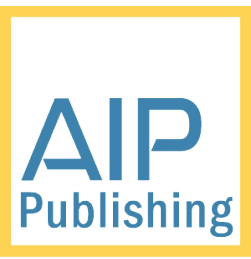

60 (a.)

This manuscript was accepted by Phys. Plasmas. Clickdere to see the version of record.
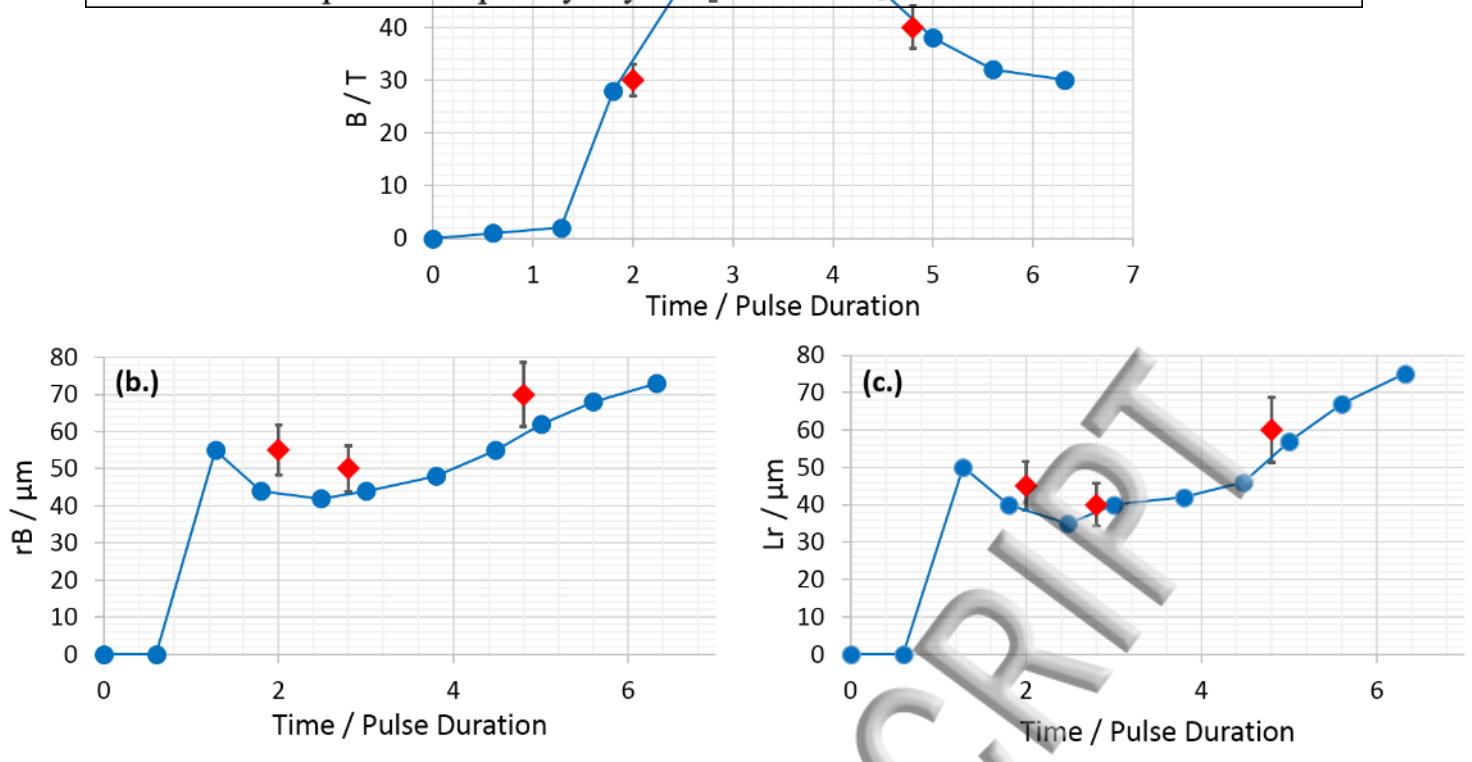

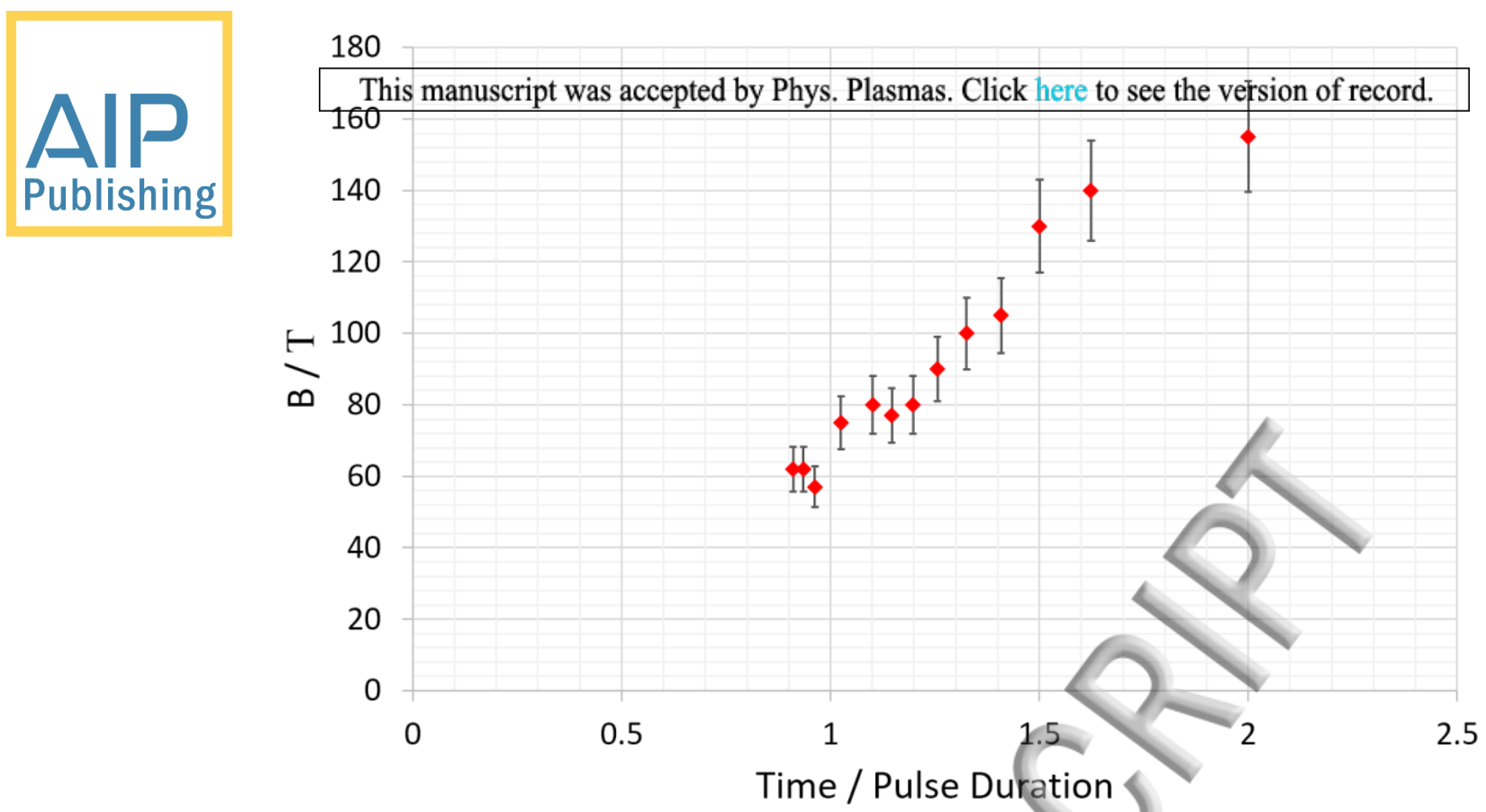